Harald Haas

\title{
Salutogenese und achtsamkeits- orientierte anthroposophische Psychotherapie am Beispiel ambulanter Gruppentherapien bei «Nervosität»
}

In diesem Beitrag soll das Konzept der Salutogenese vorgestellt und eine Anwendung in der Anthroposophischen Psychotherapie dargestellt werden.

\section{Die Entwicklung des Salutogenese-Konzepts bei Antonovsky}

Ein guter Überblick zum Konzept der Salutogenese findet sich bei der Bundeszentrale für gesundheitliche Aufklärung in Köln unter dem Titel «Was erhält Menschen gesund? Antonovskys Modell der Salutogenese Diskussionsstand und Stellenwert» [1]. In dieser Übersichtsarbeit wurde der Forschungsstand bis zum Jahr 2001 zusammengetragen. Das Salutogenese-Konzept (Gesundheitsentstehungskonzept) ist, trotz des älter klingenden Namens, ein recht neuer Begriff und wurde von Aaron Antonovsky entwickelt. Dieser veröffentlichte das Konzept erstmals 1970 unter dem Titel «Health, Stress and Coping: New Perspectives on Mental and Physical Well-Being». Später folgte das Buch «Unraveling the Mystery of Health How People Manage Stress and Stay Well» [2], das erst 1997, d.h. 10 Jahre später, beim Verlag der Deutschen Gesellschaft für Verhaltenstherapie in Tübingen unter dem Titel «Salutogenese - Zur Entmystifizierung der Gesundheit» [3] in der deutschen Übersetzung zugängig wurde.
Die medizinisch-soziologische Forschung Antonovskys entstand hauptsächlich in Israel, wohin Antonovsky, aus den USA stammend, 1960 emigrierte. Er untersuchte insbesondere, wie es den Menschen ergangen war, die ein Konzentrationslager erlebt hatten und in den neuen Staat Israel emigrierten, darauf hin, wie sich ihr gesundheitliches Befinden und Wohlbefinden entwickelt hatte. Dabei kam er zu dem sehr erstaunlichen Ergebnis, dass fast 30\% der Frauen, die er untersuchte, trotz ihrer unerträglichen Erfahrungen in einem guten Gesundheitszustand waren und sich wohl fühlten [3]. Dieses Ergebnis des relativ hohen Anteils von gesunden Überlebenden war für ihn unerwartet. Dabei stellte sich ihm die Frage, was für eine Einstellung oder Haltung diese Menschen haben mussten, damit es ihnen möglich war, ein so grauenvolles Geschehen gut überstehen zu können. Bei weiteren Forschungen zeigte sich, dass es vor allem drei Grundhaltungen sind, die gesundheitsfördernd wirken:

Die erste Grundhaltung nannte Antonovsky das Gefühl der Verstehbarkeit (sense of comprehensibility), die zweite das Gefühl von Handhabbarkeit (sense of manageability) und die dritte das Gefühl von Sinnhaftigkeit (sense of meaningfulness) [3].

Zur Verstehbarkeit führt Antonovsky aus, dass diese Komponente die Erwartung bzw. Fähigkeit von Menschen, Stimuli - auch unbekannte - als geordnete, konsistente, strukturierte
Informationen verarbeiten zu können, beschreibt. Dazu gehört auch, nicht mit Reizen konfrontiert zu sein, die chaotisch, willkürlich, zufällig oder unerklärlich sind. Dies entspricht einem sinnhaften kognitiven Verarbeitungsmuster.

Bei dem Gefühl der Handhabbarkeit heisst es: «Diese Komponente beschreibt die Überzeugung eines Menschen, dass Schwierigkeiten lösbar sind. Das Ausmass, in dem man wahrnimmt, dass man geeignete Ressourcen zur Verfügung hat, um den Anforderungen zu begegnen» [1]. Beim Gefühl der Sinnhaftigkeit ist gemeint: «das Ausmass, in dem man das Leben als emotional sinnvoll empfindet: dass wenigstens einige der vom Leben gestellten Probleme und Anforderungen es wert sind, dass man Energie in sie investiert, dass man sich für sie einsetzt und sich ihnen verpflichtet, dass sie eher willkommene Herausforderungen sind als Lasten, die man gerne los wäre» [1].

Im Salutogenese-Konzept wurden bereits bekannte psychotherapeutische Ideen angesprochen. Die Sinnfrage war ja länger zuvor besonders von Viktor Frankl [4] als bedeutend angesehen worden. Die anderen Faktoren fanden sich bereits im Stress-CopingKonzept.

Antonovsky führte die drei Grundhaltungen unter dem Begriff des Kohärenzgefühls zusammen, im Englischen «sense of coherence» (SOC). Er beschreibt das Kohärenzgefühl als

\section{KARGER}

(c) 2017 S. Karger GmbH, Freiburg

Fax +497614520714
Dr. med. Harald Haas

Psychiatrie und Psychotherapie FMH

Anthroposophische Medizin VAOAS und GAÄD

Anthroposophische Psychotherapie DtGAP

Münstergasse 68, 3011 Bern, Schweiz

harald.haasbe@bluewin.ch 
«eine globale Orientierung, die das Ausmass ausdrückt, in dem jemand ein durchdringendes, überdauerndes und dennoch dynamisches Gefühl des Vertrauens hat, dass erstens die Anforderungen aus der inneren oder äusseren Erfahrungswelt im Verlauf des Lebens strukturiert, vorhersehbar und erklärbar sind und dass zweitens die Ressourcen verfügbar sind, die nötig sind, um den Anforderungen gerecht $\mathrm{zu}$ werden. Und drittens, dass diese Anforderungen Herausforderungen sind, die Investitionen und Engagement verdienen» [1]. Zum Erfassen des Kohärenzgefühls entwickelte er einen Fragebogen, der sich aus den einzelnen Komponenten der Salutogenese zusammensetzt.

Bei der weiteren Untersuchung zu dem Konzept [1] fand man in Forschungsarbeiten, dass der Fragebogen zum Kohärenzgefühl eigentlich im umgekehrten Verhältnis das Ausmass von Ängstlichkeit oder Depressivität abbildet. Menschen, die ängstlich oder depressiv sind, haben ein sehr niedriges und Menschen, die diese Belastungen nicht zeigen, haben ein hohes Kohärenzgefühl. Dies führte zu der kritischen Frage, was überhaupt mit dem Kohärenzgefühl erfasst wird?

Antonovsky versuchte wiederholt, auch den Zusammenhang von körperlichen Krankheiten mit dem Instrument des Kohärenzgefühls erfassen zu können. Bei der Forschung darüber zeigte sich, dass gewisse Korrelationen zu finden sind, die aber meistens nur schwach ausgeprägt waren. Dagegen fanden sich insbesondere bei psychischen und psychosomatischen Krankheiten salutogenetische Zusammenhänge, was vielfach bestätigt wurde [1].

Antonovsky sieht das Kohärenzgefühl als zentralen Mittler im StressCoping-Modell, wo ein sehr kompliziertes Zusammenwirken verschiedener Komponenten von Stressoren auf der einen Seite sowie Widerstandsressourcen, d.h. Ressourcen, die befähigen, sich widrigen Situationen aussetzen zu können, und Lebenserfahrungen auf der anderen Seite entgegenstehen.
Tab. 1. Grundlegende Annahmen des pathogenetischen und salutogenetischen Modells

\begin{tabular}{|c|c|c|}
\hline Annahme in Bezug auf & Pathogenetisches Modell & Salutogenetisches Modell \\
\hline $\begin{array}{l}\text { Selbstregulierung des } \\
\text { Systems }\end{array}$ & Homöostase & $\begin{array}{l}\text { Überwindung der Heterostase } \\
\text { (Ungleichgewicht, fehlende Stabilität) }\end{array}$ \\
\hline $\begin{array}{l}\text { Gesundheits- und } \\
\text { Krankheitsbegriff }\end{array}$ & Dichotomie & Kontinuum \\
\hline $\begin{array}{l}\text { Reichweite des } \\
\text { Krankheitsbegriffs }\end{array}$ & $\begin{array}{l}\text { Pathologie der Krankheit } \\
\text { reduktionistisch }\end{array}$ & $\begin{array}{l}\text { Geschichte des Kranken und } \\
\text { seines Krank-Seins, ganzheitlich }\end{array}$ \\
\hline $\begin{array}{l}\text { Gesundheits- und } \\
\text { Krankheitsursachen }\end{array}$ & $\begin{array}{l}\text { Risikofaktoren, } \\
\text { negative Stressoren }\end{array}$ & $\begin{array}{l}\text { heilsame Ressourcen, } \\
\text { Kohärenzsinn }\end{array}$ \\
\hline Wirkung von Stressoren & potenziell krankheitsfördernd & $\begin{array}{l}\text { krankheits- und } \\
\text { gesundheitsfördernd }\end{array}$ \\
\hline Interventionen & $\begin{array}{l}\text { Einsatz wirksamer Heilmittel } \\
\text { («magic bullets», «Wunderwaffen») }\end{array}$ & $\begin{array}{l}\text { aktive Anpassung, Risikoreduktion } \\
\text { und Ressourcenentwicklung }\end{array}$ \\
\hline
\end{tabular}

Dieses Zusammenwirken bestimme das Befinden im Gesundheits-Krankheits-Kontinuum. Ob das Kohärenzgefühl, wie eben zusammengefasst, mit dem Stress-Coping-Modell in dieser Art verbunden werden kann, ist gemäss aktueller Forschung noch offen, auch wenn es theoretisch überzeugend wirkt.

Ein weiteres Thema, das durch das Salutogenese-Konzept angesprochen ist, ergibt sich durch die Gegenüberstellung des pathogenetischen Krankheitsmodells, wo eine Polarisierung von Gesundheit und Krankheit vertreten wird, zum Kontinuum zwischen Gesundheit und Krankheit beim salutogenetischen Modell nach Noack (Tab. 1) [1].

\section{Salutogenese in der anthroposophisch- medizinischen Rezeption}

Das Salutogenese-Konzept ist im Bereich der Anthroposophischen Medizin insbesondere durch die frühere Leiterin der medizinischen Sektion am Goetheanum in Dornach, Michaela Glöckler, bekannt geworden. Zunächst erschien von ihr ein Vortrag in der Schriftenreihe des Vereins, "Gesundheit aktiv», unter dem Titel «Salutogenese. Wo liegen die Quellen leiblicher, seelischer und geistiger Gesundheit?» [5]. In ihrem 2 Jahre später erschienenen Buch «Kindsein heute.
Schicksalslandschaft aktiv gestalten. Umgang mit Widerständen - ein salutogenetischer Ansatz» [6] führt sie das Konzept Antonovskys mit den Themen Heterostase, Kohärenzgefühl und Widerstandsressourcen weiter aus und bezieht es dann auf die (anthroposophischen) Gesundheitsquellen für das Kind. In der klinischen Forschung und Anwendung hat Peter F. Matthiessen von der Universität Witten/Herdecke das salutogenetische Konzept umgesetzt. Er hat das Projekt für einen Qualitätszirkel mit niedergelassenen Ärzten gestartet, wobei die Praxistätigkeit unter dem salutogenetischen Gesichtspunkt vertieft betrachtet wurde. Die Erfahrungen damit wurden, neben den konzeptuellen Grundlagen, von Ottomar Bahrs und Peter F. Matthiesen als Herausgeber unter dem Titel «Gesundheitsfördernde Praxen. Die Chancen einer salutogenetischen Orientierung in der hausärztlichen Praxis» [7] veröffentlicht.

\section{Der salutogenetische Gedanke bei Rudolf Steiner - Selbstentwicklung und Willensschulung}

Vortragsausführungen Rudolf Steiners über die Selbsterziehung des Menschen können als relevant für das Thema der Salutogenese angesehen werden. Man kann wohl davon ausgehen, dass die Menschen, die ein ausge- 
prägtes Kohärenzgefühl haben, dies nicht nur durch Erziehung von aussen, sondern auch in der Auseinandersetzung mit der Welt, d.h. durch Selbsterziehung bis zum Erwachsenenalter, erworben haben. Antonovsky ging davon aus, dass das Kohärenzgefühl in der Kindheit und Jugend entwickelt würde, was aber in dieser Einschränkung schon kritisiert wurde [1].

Als Grundelemente der Selbsterziehung tauchen bei Rudolf Steiner die Seelenqualitäten von Staunen, Mitgefühl und Gewissen auf, worüber er am 27.12.1911 in Hannover sprach [8]. Er wies dabei mit dem Staunen, der Verehrung, dem weisheitsvollen Einklang mit den Welterscheinungen bis zum Ergeben in das Weltenganze auf die Qualitäten hin, die zur Wahrhaftigkeit führen können. Im folgenden 2. Vortrag des Hannoverschen Vortragszyklus [8] nannte er diese Qualitäten Elemente der Willensschulung, was auch wiederum auf die Selbsterziehung hinweist.

Am 14. März 1912 hat Rudolf Steiner Aspekte der Willensschulung in einem öffentlichen Vortrag in Berlin mit dem Titel «Die Selbsterziehung des Menschen im Lichte der Geisteswissenschaft» [9] aufgegriffen. Als die Bereiche der Willensschulung nannte Rudolf Steiner hier das Gewissen, das Mitleid oder die Liebefähigkeit sowie die Aufmerksamkeit, die Andacht oder das Erfassen von Grundgedanken als Schulung der Vorstellung. Der Begriff des Gewissens ist hier nicht im Sinne der vergangenheitsorientierten Assoziation von Schuldgefühlen gemeint, sondern ist als Mittel zur voraussehenden, die freie Entscheidungsfähigkeit zu einer Tat mit einschliessenden Qualität zu verstehen. Damit erhält es mehr noch die Qualität des Gefühls der Sinnhaftigkeit des Kohärenzgefühls der Salutogenese. Das Mitleid ist auch bei Antonovsky die Voraussetzung für das Gefühl der Handhabbarkeit, da der Mensch nicht nur auf seine eigenen Fähigkeiten zu einer Problemlösung bauen kann, sondern immer auch auf die Unterstützung anderer angewiesen ist, was das Mitleid voraussetzt. Aufmerksamkeit und Andacht können als die Grundelemente des Gefühls der Verstehbarkeit angesehen werden, sodass insgesamt die Steiner'schen Aspekte der Willensschulung den Elementen des Kohärenzgefühls als entsprechend verstanden werden können.

Gegen Ende des Vortrags über «die Selbsterziehung» [9] beschreibt Steiner die Nervosität als «falsche Erziehung des Willens». Rudolf Steiner betont, dass der Wille im Leben nur durch die Auseinandersetzung mit dem Leben und nicht durch intellektuelle Methoden geschult werden könne, die nur auf das Vorstellungsleben wirken. Bereits Anfang des Jahres 1912, am 11. Januar in Berlin, hatte Rudolf Steiner Übungen zur Überwindung der Nervosität unter dem Titel «Nervosität und Ichheit» [10] in einem Vortrag angegeben.

\section{Der Vortrag «Nervosität und Ichheit» [10]}

Schon im Inhaltsüberblick wird deutlich, dass neben verschiedenen Arten der Nervosität, wie «Hast des Seelenlebens, Entschlussunfähigkeit und Nachahmung organischer Krankheiten», auch die Ursachen der Nervosität in der «Schwächung des Ätherleibes (Lebensleibs) durch die Zeitkultur und durch Treiben von Dingen ohne Interesse» stichpunktartig aufgeführt sind. Es folgen dann die Übungen,

(1) «bewusstes Verlegen von Gegenständen»,

(2) «bewusstes Verändern von Schreibgewohnheiten",

(3) «Rückwärtsdenken»,

(4) «Beobachtung der eigenen Gebärden; bewusstes $<$ Anders-als-sonstTun e einiger Dinge, z.B. Benutzung der linken Hand»,

(5) «Versagen, Unterdrücken kleiner Wünsche»,

(6) «eigenes Erwägen des Für und Wider einer Sache»,
(7) «Zurückhaltung im Urteilen, besonders bei eigener Betroffenheit».

Aus dem Bearbeiten des Vortragstextes von «Nervosität und Ichheit» ergibt sich die Frage, welche Verbindungen die sieben verschiedenen Übungen zu den anfangs im Vortrag erscheinenden Arten der Nervosität haben. Dabei kann auffallen, dass die erste Übung direkt an die Darstellung der «leichten Vergesslichkeit» anknüpft. Geht man nun von diesem zentralen Abschnitt nicht nur nach vorne weiter zu den folgenden Übungen, sondern auch zurück zu den Schilderungen der anderen Arten von Nervosität, findet man einen inneren Zusammenhang der weiteren Übungen mit bestimmten Formen der Nervosität. Dabei treffen dann zusammen: - das «bewusste Verändern derSchreibgewohnheiten» mit der «Bedeutung des Zusammenhangs zwischen Innerstem Wesenskern und Tätigkeit» und der Schilderung von dem, dass es «für die gesamte Wesenheit des Menschen kaum etwas Schlimmeres (gibt), als wenn man seelisch, mit seinem Herzen dem fern steht, was der Kopf treiben muss»,

- das «Rückwärtsdenken» von Handlungsabläufen und die Schädlichkeit der «Einpaukerei»,

- das «Beobachten der eigenen Gebärden» und das erwähnte «nervöse Treiben in dem öffentlichen Leben», das Rudolf Steiner als «politischer Alkoholismus» bezeichnet,

- die «Beherrschung des astralischen Leibes (Seele) durch das Ich mit Hilfe der Willenskultur» und die «Krankheitsformen ... für die man eigentlich keine organischen Ursachen angeben kann, die aber zuweilen organische Krankheiten in einer täuschenden Weise nachbilden»,

- das «Erwägen des Für und Wider einer Sache», und "dass die Menschen ... gegenüber Dingen, bei denen sie zu Entschlüssen kommen sollten, nicht zu Entschlüssen vorrücken können», und schliesslich, 
- dass die «Zurückhaltung im Urteilen» mit der geschilderten Nervositätsform des «seelischen Zappelfritzen», der «unvermögend ist, einen Gedanken ordentlich festzuhalten und ihn wirklich in seinen Konsequenzen $\mathrm{zu}$ verfolgen» $\mathrm{zu}-$ sammenfällt.

Die oben angeführten Zusammenhänge der Vortragsthemen zeigen, dass die zu Beginn des Vortrags geschilderten sieben Formen oder Arten der Nervosität in der Anordnung gespiegelt zusammenhängen mit den sieben Übungen des zweiten Vortragsteils.

\section{Vergleich der Formen der Nervosität aus dem Vortrag «Nervosität und Ichheit» mit dem achtgliedrigen Pfad des Gautama Buddha}

Vergleicht man die Prinzipien zur Ausbildung des achtgliedrigen Pfades [11] (Tab. 2), die heute als die Qualitäten der Achtsamkeit angesehen werden, mit den Formen der Nervosität aus «Nervosität und Ichheit», so findet man eine weitgehende Übereinstimmung mit den Themen, die angesprochen werden. Diese sind in der gleichen Reihenfolge aufgeführt: 1 . «Gedanken nicht ordentlich festhalten und verfolgen können», 2. «nicht zu Entschlüssen vorrücken können», 3 . «Nervosität in Krankheitsformen, die zuweilen organische Krankheiten in einer täuschenden Weise nachbilden», 4. «öffentliches nervöses Gebaren», 5. «ein geringes Band von Interesse verbindet ... den menschlichen Seelenkern mit dem, was die Menschen annehmen», 6. «eine geringe Verbindung ... zwischen den menschlichen Seelenkern und demjenigen, was der Mensch treibt» sowie 7. die «leichte (häufige) Vergesslichkeit». Was im Vortrag «Nervosität und Ichheit» nicht so offensichtlich erwähnt wird, ist der 8. Punkt der Besinnung. Diesen kann man jedoch zu Beginn und am Ende des Vortrags sowie in einigen

Tab. 2. Planetenaspekte der Wochentage (nach [11])

\begin{tabular}{ll}
\hline Samstag & «Vorstellungen, die richtige Meinung» (Saturn) \\
Sonntag & «sich entschliessen, richtiges Urteil» (Sonne) \\
Montag & «das Reden, das richtige Wort» (Mond) \\
Dienstag & «die äusseren Handlungen, die richtige Tat» (Mars) \\
Mittwoch & «die Einrichtung des Lebens, richtiger Standpunkt» (Merkur) \\
Donnerstag & «das menschliche Streben, alle vorangegangenen Übungen zur Gewohnheit \\
Freitag & werden lassen» (Jupiter) \\
\hline Zusammenfassung & «vom Leben lernen, das richtige Gedächtnis» (Venus) \\
\hline
\end{tabular}

Zwischenbemerkungen finden, wo die Bedeutung des Verständnisses der Wesensglieder des Menschen (physischer Leib, Ätherleib, Astralleib und Ich [12]) für das menschliche Leben und die Wirkungen der Übungen gegen die Nervosität beschrieben werden. Die thematische Übereinstimmung der acht Punkte der «Ausbildung des achtgliedrigen Pfades» in der Beschreibung der «Übungen für die Tage der Woche» (Tab. 2), mit den typischen Formen der Nervosität, dürfte aus der Formulierung sonst durchwegs einleuchtend sein, mit Ausnahme des 3. Punktes. Dort bildet die Sinnverwandtschaft, dass die «Unterdrückung von Wünschen, wenn die Nichtausführung der Wünsche keinen Schaden bringt» im Bereich des Redens zu finden ist, die Brücke.

In der Beschreibung der Übungen «für die Tage der Woche» [11], die dem achtgliedrigen Pfad des Gautama Buddha entsprechen, heisst es: «Der Mensch muss auf gewisse Seelenvorgänge Aufmerksamkeit und Sorgfalt verwenden, die er gewöhnlich sorglos und unaufmerksam ausführt. Es gibt acht solche Vorgänge.»

Bestechend in der Formulierung ist der Hinweis auf die Sorglosigkeit und Unaufmerksamkeit, die heute zu den wesentlichen Symptomen der Aufmerksamkeitsdefizitstörung gerechnet werden, sodass eine gewisse Übereinstimmung des Steiner'schen Nervositätsbegriffs mit der aktuellen Diagnose gesehen werden kann.

Durch Rudolf Steiners Zuordnung der Übungselemente zu den Wochentagen ergeben sich hier sehr interes- sante Bezüge zu den Planetenaspekten der Wochentage (Tab. 2).

Aus diesen Zusammenhängen ergeben sich Möglichkeiten, den Formen der Nervosität Planetenprozesse zuordnen zu können und auch die entsprechenden Vokal- oder Planetengesten aus der Eurythmie bzw. Heileurythmie zu finden.

\section{Ein «anthroposophischer Achtsamkeitskurs» nach «Nervosität und Ichheit»}

In meiner therapeutischen Tätigkeit zeigte sich, dass es für die Patienten meistens ungewohnt ist und es ihnen zunächst schwerfällt, selbstständig die Übungen $\mathrm{zu}$ machen. So braucht es nach einem Entschluss dafür häufig die Unterstützung durch Nachfragen oder auch durch gemeinsames Probieren. Deshalb ergab sich die Frage, die Übungen in einer Gruppe zu erarbeiten und sich gegenseitig von den Erfahrungen beim Üben zu berichten. Die Struktur des Gruppenprogramms ist dabei angelehnt an das Konzept von Kabat-Zinn in der Form der «Mindfulness-based stress reduction» [13] und von psychologischer Seite in der «Mindfulness-based cognitive therapy for depression» [14], die seit einigen Jahren entwickelt und erprobt wurden. Dabei wird die Therapie einmal wöchentlich über einen Zeitraum von 8 Wochen abgehalten, und die Teilnehmer üben nach Möglichkeit jeden Tag alleine die neu gelernten Elemente. Die einzelnen Körperwahrnehmungs-, Beobachtungs- 
Tab. 3. Tabellarische Übersicht über das Programm

\begin{tabular}{|c|c|c|c|c|}
\hline Woche & Thema & $\begin{array}{l}\text { Übung (vor allem R. Steiner, } \\
\text { «Nervosität und Ichheit») }\end{array}$ & Heileurythmie & $\begin{array}{l}\text { Nervosität, Seelenschwäche, } \\
\text { Krankheit }\end{array}$ \\
\hline 1 & $\begin{array}{l}\text { Der menschliche Leib } \\
\text { Die vier Elemente }\end{array}$ & $\begin{array}{l}\text { • Elemente-Übung } \\
\text { • Verlegen von Gegenständen }\end{array}$ & $\begin{array}{l}\text { •A-Gebärde } \\
\text { • H-A Lachen } \\
\text { •A-H Verehrung }\end{array}$ & - Vergesslichkeit \\
\hline 2 & $\begin{array}{l}\text { Die Seele } \\
\text { Die Temperamente }\end{array}$ & $\begin{array}{l}\text { - Handschrift ändern } \\
\text { - Was ist mir heute leicht-/ } \\
\text { schwergefallen }\end{array}$ & $\begin{array}{l}\text { - Licht } \\
\text { - Schwere } \\
\text { - O-Gebärde }\end{array}$ & $\begin{array}{l}\text { - Geringe Verbindung zwischen } \\
\text { Seelenkern und Tun } \\
\text { - Burnout } \\
\text { - Schreckhaftigkeit und Ängstlichkeit } \\
\text { - Verkrampfungen }\end{array}$ \\
\hline 3 & Das Ich & $\begin{array}{l}\text { - Rückwärts denken } \\
\text { - Selfing }\end{array}$ & $\begin{array}{l}\text { - I-A-O i.d. Gestalt } \\
\text { - I-Gebärde } \\
\text { - Ich bin Da }\end{array}$ & $\begin{array}{l}\text { - Desinteresse } \\
\text { - Selbstzweifel und Überbesorgtheit }\end{array}$ \\
\hline 4 & Der Geist & $\begin{array}{l}\text { - Sich selbst beobachten } \\
\text { - Bewegungsgewohnheiten } \\
\text { ändern }\end{array}$ & $\begin{array}{l}\cdot \text { E-Gebärde } \\
\text { - Grosses E } \\
\text { - Liebe - E } \\
\text { - Fünfstern }\end{array}$ & $\begin{array}{l}\text { - Politischer Alkoholismus } \\
\text { - Überaktivität und Kontrollverlust } \\
\text { - Zorn und Scham }\end{array}$ \\
\hline 5 & Mitgefühl & $\begin{array}{l}\text { - Verzicht auf die Erfüllung } \\
\text { kleiner Wünsche } \\
\text { - Wo erlebte ich heute Mitgefühl? }\end{array}$ & $\begin{array}{l}\text { - M-Schritt } \\
\text { - Sympathie } \\
\text { - Antipathie }\end{array}$ & $\begin{array}{l}\text { - Psychosomatische Erkrankungen } \\
\text { - Abhängigkeit von } \\
\text { Ersatzbefriedigungen }\end{array}$ \\
\hline 6 & Gewissen & $\begin{array}{l}\text { - Entscheidungen abwägen und } \\
\text { durchführen } \\
\text { - Wo erlebte ich heute Gewissen? }\end{array}$ & $\begin{array}{l}\text { - Rhythmus } \\
\text { - Ja-Nein }\end{array}$ & $\begin{array}{l}\text { - Entschlusslosigkeit, mit sich selbst } \\
\text { nichts anzufangen wissen } \\
\text { - Handlungszwänge }\end{array}$ \\
\hline 7 & $\begin{array}{l}\text { Staunen } \\
\text { Pflanzenbetrachtung }\end{array}$ & $\begin{array}{l}\text { - Bewusste Meinungsbildung } \\
\text { - Verzicht auf Kritik } \\
\text { - Wo erlebte ich heute Staunen? }\end{array}$ & $\begin{array}{l}\text { - Hinterer Raum } \\
\text { - Hoffnung - U }\end{array}$ & $\begin{array}{l}\text { - Gefangensein in Vorstellungen } \\
\text { - Gedankenzwang }\end{array}$ \\
\hline 8 & $\begin{array}{l}\text { Umkehrung des } \\
\text { Übungsprogramms }\end{array}$ & - Andacht, Mitgefühl, Gewissen & $\begin{array}{l}\text { - A-Staunen } \\
\text { - O-Mitgefühl } \\
\text { - I- Gewissen }\end{array}$ & $\begin{array}{l}\text { - Zweifel an der Wirksamkeit geistiger } \\
\text { Anschauungen } \\
\text { - Unselbstständigkeit }\end{array}$ \\
\hline
\end{tabular}

und Bewegungsübungen stehen alle unter dem Gesichtspunkt des nicht urteilenden Erkennens, das als Grundhaltung der Achtsamkeit angenommen wird.

Für einen «Anthroposophischen Achtsamkeitskurs» konnten statt den «Körpermeditationen» und YogaÜbungen Eurythmie bzw. Heileurythmie-Elemente eingesetzt werden. Die Idee dazu wurde erstmals im Sammelband «In der Nacht sind wir zwei Menschen - Arbeitseinblicke in die anthroposophische Psychotherapie» [15] im Beitrag «Selbsterziehung und der achtgliedrige Pfad» veröffentlicht. Zum Einbezug der Eurythmie hat noch der oben genannte öffentliche Vortrag Rudolf Steiners, «Die Selbsterziehung des Menschen im Lichte der Geisteswissenschaft», angeregt $[9,11]$. Dort heisst es: «wenn der Mensch da- gegen im turnerischen Spiel, in gymnastischen Übungen sich betätigt, (...) dann haben wir es mit einem selbsterzieherischen Spiel zu tun.» Dies kann doch gut auf die Eurhythmie bezogen werden.

In den letzten Jahren konnte der Anthroposophische Achtsamkeitskurs in der Zusammenarbeit mit dem Heileurythmisten Theodor Hundhammer bei inzwischen 7 Gruppen von 6-10 Teilnehmern in der eigenen Praxis erprobt wurden. Die Gruppenarbeit begann jeweils nach einer Einführung mit einem Rückblick auf das Üben der vergangenen Woche, wo sich die meisten Kursteilnehmer über ihre individuellen Erfahrungen äusserten. Der Austausch über das eigene Üben wirkte zusätzlich motivierend und auch entlastend gegenüber den eigenen Ansprüchen an das Üben. Die
Heileurythmie-Übungen wurden als kraftspendend und wirkungsvoll erlebt. Die Übungen von «Nervosität und Ichheit» [10] brachten einige Selbsterkenntnis-Momente zutage, was $\mathrm{zu}$ anregenden Diskussionen in der Gruppe führte. Die Übungen wurden unterschiedlich intensiv praktiziert; vielfach wurden sie den eigenen Bedürfnissen und Möglichkeiten angepasst. Zum Teil konnten Übungselemente direkt im Alltag angewendet werden. Auch ohne die Übungsaufgaben fortlaufend zu praktizieren, konnte eine Erleichterung in Alltagssituationen wahrgenommen werden.

Der Kurs zur Nervosität dient insbesondere zur Vorbeugung und Verbesserung von Aufmerksamkeits- und Gedächtnisproblemen, Zweifel und Entscheidungsschwäche, Burnout und depressiven Verstimmungen, 
Ängsten, Hypersensitivität und psychosomatischen Beschwerden.

Diesen Symptomen der neurotischen Belastungsstörungen und somatoformen Störungen liegt eine innere Struktur zugrunde, die früher unter dem Begriff Nervosität oder nervöse Störung zusammengefasst wurden. Sie treten heutzutage immer häufiger im Sinne einer «Volkskrankheit» auf, wobei das Alltagsleben der davon Betroffenen stark beeinträchtigt sein kann. Deshalb sollten neue Wege der Prävention und Therapie dafür gesucht werden, wie sie im Gruppenkurs aufgezeigt werden.

\section{Das Kursprogramm in der Übersicht}

Die Übersicht über das Kursprogramm (Tab. 3) zeigt, dass noch weitere Themen (erste Spalte) eingefügt wurden. Dies sind zum einen die sogenannten vier Wesensglieder psychischer und ätherischer Leib und Elemente, die Seele und die Temperamente, das Ich und der Geist [12], die Rudolf Steiner in dem Vortrag erwähnt, da diese Themen selbstverständlich nicht als geläufig angesehen werden. Zum anderen sind es die Seelenstimmungen von Mitgefühl, Ge- wissen und Staunen, wie sie oben schon als salutogenetische Elemente hergeleitet wurden. Auch bei den «Nervositätsübungen» finden sich Ergänzungen, die sich aus der jeweiligen Thematik ergeben. Neu entwickelt wurde auch die Zuordnung der (Heil-) Eurythmieübungen, die sich im Laufe der Gruppenanlässe konkretisierte. Die vierte Spalte zeigt die seelischen Auffälligkeiten, mit denen die jeweiligen Übungen in Beziehung stehen können. Dies ermöglicht es auch, eine symptomorientierte Auswahl bei einer Einzelanwendung treffen zu können.

\section{Weitere geplante Auswertungen und Anwendungen}

Neben den konkreten Erfahrungen und Rückmeldungen der bisherigen Teilnehmerinnen ist mit der Unterstützung von Prof. Dr. Ursula Wolf, Anthroposophische Medizin, Co-Direktorin des Instituts für Komplementäre Medizin in Bern, eine testpsychologische Auswertung im Gang, aber derzeit noch nicht abgeschlossen. Dabei wird beobachtet, inwieweit der Salutogenese-Fragebogen von Antonovsky und Coping-Fragebogen die
Veränderungen durch den Übungsprozess widerspiegeln können. Zur Beurteilung des Befindens wurde bei einzelnen Patienten auch der Mehrdimensionale Befindlichkeitsfragebogen angewandt. Als physiologischen Parameter für eine Prä- und Postbeurteilung des vegetativen Befindens wurde damit begonnen, die Herzratenvariabilität zu erheben. Aus einzelnen Resultaten ist bisher ersichtlich, dass testpsychologische Veränderungen nur auftraten, wenn zuhause regelmässig geübt wurde, was nicht immer der Fall war, sodass allein die Teilnahme an der Gruppe keine wesentlichen Veränderungen bewirkte.

Das Dossier des gesamten Kursprogramms mit den Texten des Vortrags «Nervosität und Ichheit» [10] steht unter www.achtsamwerden.ch zur freien Verfügung.

Sicher ist denkbar, dass statt der Eurythmieübungen auch andere Künste im Übungsablauf integriert werden könnten, was in Schulungsgruppen auch schon mit der therapeutischen Sprachgestaltung und der Musiktherapie erprobt werden konnte. Bei Interesse kann das Kursprogramm auch auf Mailanfrage (theodor@bewegteworte.ch) als Word-Datei zugesendet werden, um die eigenständige Bearbeitung zu erleichtern.

\section{Literatur}

1 Bengel J (Hrsg): Was erhält Menschen gesund? Antonovskys Modell der Salutogenese - Diskussionsstand und Stellenwert. Forschung und Praxis der Gesundheitsförderung. Band 6. Köln, Bundeszentrale für gesundheitliche Aufklärung, 2001. www.bzga.de.

2 Antonovsky A: Unraveling the Mystery of Health - How People Manage Stress and Stay Well. San Francisco, Jossey-Bass, 1987.

3 Antonovsky A: Salutogenese - Zur Entmystifizierung der Gesundheit. Tübingen, DGVT, 1997.

4 Frankl VF: Der leidende Mensch. Anthropologische Grundlagen der Psychotherapie. Bern, Huber, 1996.

5 Glöckler M: Salutogenese. Wo liegen die Quellen leiblicher, seelischer und geistiger Gesundheit? Bad Liebenzell, Verein für ein Anthroposophisches Heilwesen, 2007.

6 Glöckler M: Kind sein heute. Schicksalslandschaft aktiv gestalten. Umgang mit Widerständen - ein salutogenetischer Ansatz. Stuttgart, Johannes M. Mayer, 2003.

7 Bahrs O, Matthiessen PF (Hrsg): Gesundheitsfördernde Praxen. Die Chancen einer salutogenetischen Orientierung in der hausärztlichen Praxis. Bern, Huber, 2007.

8 Steiner R: Die Welt der Sinne und die Welt des Geistes. GA-Nr. 134. Hannover 27. und 28. Dezember 1911. Dornach, Rudolf Steiner, 1990.

9 Steiner R: Menschengeschichte im Lichte der Geistesforschung. GA-Nr. 61. Berlin, 14. März 1912. Dornach, Rudolf Steiner, 1987.

10 Steiner R: Nervosität und Ichheit - Stressbewältigung von Innen. Vortrag vom 11.1.1912, eingeleitet von Frank Meyer, Sonderdruck aus Bibliographie-Nr. 143. Dornach, Rudolf Steiner, 1994.
11 Haas H (Hrsg): Steiner R: Sich selbst erziehen Das Geheimnis der Gesundheit, ed 2. Dornach, Rudolf Steiner, 2014.

12 Steiner R: Theosophie, ed 33. Dornach, Rudolf Steiner, 2013.

13 Kabat-Zinn J: Mindfulness-Based Stress Reduction (MBSR). 2005.

14 Segal ZV, Williams JMG, Teasdale JD: Mindfulness-Based Cognitive Therapy for Depression (MBCT). New York, NY, Guilford Press, 2008.

15 Reiner J (Hrsg): In der Nacht sind wir zwei Menschen - Arbeitseinblicke in die anthroposophische Psychotherapie. Stuttgart, Freies Geistesleben, 2012. 